\title{
Checklist das alterações de audição e linguagem em idosos senescentes
}

\author{
Checklist of hearing and language disorders in elderly senescent \\ Lista de verificación de trastornos de audición y lenguaje en los ancianos senescentes
}

Recebido: 04/04/2021 | Revisado: 12/04/2021 | Aceito: 15/04/2021 | Publicado: 30/04/2021

\author{
Ilanna Cibele Delgado de Araújo Fonsêca \\ ORCID: https://orcid.org/0000-0003-1786-7834 \\ Universidade Federal da Paraíba, Brasil \\ E-mail: ilannadelgado@gmail.com \\ Eva Carolina Fonsêca de Resende Cruz \\ ORCID: https://orcid.org/0000-0003-0440-4013 \\ Universidade Federal da Paraíba, Brasil \\ E-mail: ecarolinacruz@hotmail.com \\ Simone Pereira Lins Chaves \\ ORCID: https://orcid.org/0000-0002-0288-0721 \\ Universidade Federal da Paraíba, Brasil \\ E-mail: splfono@msn.com \\ Clarissa Madruga Holanda \\ ORCID: https://orcid.org/0000-0002-4191-9764 \\ Universidade Federal da Paraíba, Brasil \\ E-mail: clarissafono@ hotmail.com \\ Emerson Soares Pontes \\ ORCID: https://orcid.org/0000-0002-8455-016X \\ Universidade Federal da Paraíba, Brasil \\ E-mail: emerson-soares21@hotmail.com \\ Wagner Teobaldo Lopes de Andrade \\ ORCID: https://orcid.org/0000-0002-8600-2327 \\ Universidade Federal da Paraíba, Brasil \\ E-mail: wagner_teobaldo@yahoo.com.br \\ Ana Karênina de Freitas Jordão do Amaral \\ ORCID: https://orcid.org/0000-0002-7470-7717 \\ Universidade Federal da Paraíba, Brasil \\ E-mail: akfjafono@hotmail.com
}

\begin{abstract}
Resumo
Objetivo: elaborar um checklist para rastreamento das alterações de audição e de linguagem de idosos, por profissionais não-fonoaudiólogos. Métodos: pesquisa metodológica de abordagem quantitativa, realizada no período de dezembro/2018 a janeiro/2019, inicialmente com um grupo de experts para $1^{\text {a }}$ versão do checklist e posteriormente com um grupo de profissionais não-fonoaudiólogos para $2^{\mathrm{a}}$ versão do checklist, por meio de um instrumento de avaliação previamente elaborado. A análise foi realizada utilizando-se o índice de validação do conteúdo. Resultados: a primeira versão do checklist foi avaliada por 10 participantes do grupo de experts dando origem a $2^{\circ}$ versão que foi avaliada por 30 participantes do grupo de profissionais nãofonoaudiólogos, categorizadas e alocadas em planilha digital e variáveis analisadas de forma descritiva. Foi considerado o índice de validação do conteúdo maior ou igual a 0,78 , empregando a escala Likert como método de pontuação, dando origem a $3^{\mathrm{a}}$ versão do checklist. Conclusão: o checklist foi criado e validado nesta etapa inicial. É um instrumento de linguagem fácil e de aplicação simples. Segue para as demais etapas de validação.
\end{abstract}

Palavras-chave: Idoso; Presbiacusia; Fonoaudiologia; Comunicação; Linguagem; Audição.

\begin{abstract}
Objective: to prepare a checklist for the screening of hearing and speech disorders of elderly people by nonspeech-language pathologists. Methods: methodological research of a quantitative approach, carried out from December 2018 to January 2019, initially with a group of experts for the $1^{\text {st }}$ version of the checklist and later with a group of non-speech therapists for the $2^{\text {nd }}$ version of the checklist, by means of a previously elaborated evaluation instrument. The analysis was performed through the content validation index grater than or equal to 0.78 . Results: the first version of the checklist was evaluated by 10 participants of the expert group, originating the 2nd version evaluated by 30 participants of the group of non-speech therapists, categorized and allocated on digital spreadsheet and variables analyzed descriptively. The content validation index was considered greater than or equal to 0.78 , using the Likert scale as the scoring method, ending with the $3^{\text {rd }}$ version of the checklist. Conclusion: the checklist was created and validated in this initial step. It is an easy language tool and simple application. Go to the other validation steps.
\end{abstract}


Keywords: Aged; Presbycusis; Speech; Language and hearing sciences; Communication; Language; Hearing.

\begin{abstract}
Resumen
Objetivo: elaborar una lista de verificación para realizar un seguimiento de los trastornos auditivos y del lenguaje en los ancianos, por parte de no logopedas. Métodos: investigación metodológica con enfoque cuantitativo, realizada de diciembre / 2018 a enero / 2019, inicialmente con un grupo de expertos para la 1a versión de la lista de verificación y posteriormente con un grupo de no logopedas para la 2a versión de la lista de verificación, mediante un instrumento de evaluación previamente elaborado. El análisis se realizó mediante el índice de validación de contenido. Resultados: la primera versión del checklist fue evaluada por 10 participantes del grupo de expertos dando lugar a la segunda versión la cual fue evaluada por 30 participantes del grupo de no logopedas, categorizados y asignados en una hoja de cálculo digital y las variables analizadas de manera descriptiva. Se consideró el índice de validación de contenido mayor o igual a 0,78, utilizando la escala Likert como método de puntuación, dando lugar a la tercera versión del checklist. Conclusión: la lista de verificación fue creada y validada en esta etapa inicial. Es un instrumento fácil de usar y simple de usar. Continúe con los otros pasos de validación.
\end{abstract}

Palabras clave: Anciano; Presbiacusia; Terapia del lenguaje; Comunicación; Idioma; Audición.

\title{
1. Introdução
}

Checklists são utilizados há anos em várias ciências e situações específicas. Por exemplo, o checklist de segurança cirúrgica pré e pós-operatório é mais uma estratégia na promoção da segurança do paciente, pois possibilita monitorar sinais e sintomas preditivos de complicações cirúrgicas e detecção precoce de eventos adversos (Azevedo, Santos, \& Costa, 2015; Alpendre et al., 2017). Podem ser considerados como um passo importante para mapear o que é conhecido e, para isso, exige a organização dos fatos e do pensamento. Podem ser entendidos como um método de avaliação rápida e eficaz na sua utilização, por ser lista de itens que podem aparecer na forma de questões ou ações a serem realizadas. Podem ainda apresentar um sistema de pontuação, coletar comentários qualitativos e/ou considerar listas de verificações por meio das quais os profissionais, não necessariamente especialistas, diagnosticam problemas (Azevedo et al., 2015; Borges, Labanca, Couto, \& Guarisco, 2016).

Geralmente, o desenvolvimento de um checklist segue etapas, como: 1- concentrar-se no checklist, 2- fazer uma lista de prováveis checkpoints, 3- classificar e ordenar os checkpoints, 4- definir e detalhar as categorias, 5 determinar a ordem das categorias, 6- obter comentários iniciais do checklist, 7- rever o conteúdo do checklist, 8delinear e formatar o checklist, 9- avaliar o checklist, 10- finalizar o checklist, 11- aplicar e disseminar o checklist, 12- periodicamente analisar e rever o checklist. Dessa forma, será possível verificar seu uso, e se está adequado para aquilo a que se dirige (Azevedo et al., 2015).

Um checklist deve ser o menor e mais claro possível - idealmente de 5 a 9 pontos concisos em cada segmento, cada um dos quais verificável num minuto, e sua verificação deve ser verbal ainda que com a colocação de sinais no seu respectivo local. O seu uso mostra a terceiros não só a responsabilidade e seriedade, mas ajuda também a criar uma atmosfera de disciplina e a facilitar na concentração durante todos os procedimentos (Azevedo et al., 2015; Kim, Na, Kim, Han, \& Kim, 2016).

Serve para desenvolver melhor estratégia de segurança e são considerados soluções inovadoras, procedimentos ou programas que contribuem para reduzir e melhorar a segurança do paciente e que, simultaneamente, acrescentam valor dentro de uma perspectiva clínica e socioeconômica. Por isso, os protocolos ou checklist são usados como ferramentas úteis, e devem possuir uma boa e formal qualidade, visando a garantir resultados satisfatórios e a segurança do paciente em questão (Giannattasio \& Taniguchi, 2016; Porto, 2014).

Muitas vezes, os profissionais da área da saúde e que lidam diretamente com pessoas idosas em unidades básicas de saúde (UBS), deparam-se com dificuldades para identificar a necessidade de rastrear alterações e, 
principalmente, encaminhar aos profissionais adequados. Nesse sentido, apesar de serem encontrados em outras ciências, checklists ainda são pouco utilizados em Fonoaudiologia, e precisam ser considerados. Foi entendendo essa realidade local que surgiu a necessidade de se criar um instrumento que pudesse rastrear alterações de audição e linguagem de idosos, a fim de serem devidamente encaminhados para profissionais de Fonoaudiologia.

Portanto, o objetivo deste trabalho foi elaborar um checklist para rastrear as alterações de audição e linguagem de idosos, podendo ser aplicado por profissionais de saúde não-fonoaudiólogos, para o devido encaminhamento sempre que necessário.

\section{Metodologia}

A presente pesquisa foi norteada pelas Diretrizes e Normas Regulamentadoras dispostas na Resolução 466/2012 do Conselho Nacional de Saúde, que trata de pesquisas envolvendo seres humanos no cenário brasileiro e foi aprovada pelo Comitê de Ética em Pesquisa do Centro de Ciências da Saúde da Universidade Federal da Paraíba UFPB, sob parecer de número: 2.190.153.

Trata-se de uma pesquisa metodológica de abordagem quantitativa (Estrela, 2018). Anteriormente a este trabalho foi realizada uma revisão integrativa (já publicada) no período de fevereiro a março de 2018, na base de dados Medline, por meio do buscador PubMed e nas bibliotecas virtuais/repositórios bibliográficos Scielo, LILACS e Science Direct. Foram encontrados 13 artigos a partir dos critérios estabelecidos para busca, e cujos conteúdos (dados empíricos) deram origem aos itens da $1^{\mathrm{a}}$ versão do checklist

A amostra deste estudo foi constituída por dois grupos: GE (grupo de experts) e GP (grupo de profissionais não-fonoaudiólogos), ambos selecionados de forma intencional. Os critérios de inclusão do GE foram: fonoaudiólogos, com titulação de doutor, experts na área de estudo, docentes ou não, que tenham atividade assistencial ou de ensino, pesquisa e/ou extensão em audição e linguagem, de ambos os sexos. Para o segundo grupo foram estabelecidos os seguintes critérios de inclusão: profissional de saúde não fonoaudiólogo, com no mínimo 1 ano de prática assistencial na atenção básica junto a pessoas idosas, e atuantes nos serviços de saúde. Como critério de exclusão para ambos os grupos foi estabelecido o preenchimento incompleto do instrumento de coleta de dados.

A coleta de dados foi iniciada com os participantes do GE, que, aceitando participar do estudo, avaliaram, por meio de instrumento previamente elaborado, a $1^{\text {a }}$ versão do checklist construída após levantamento de dados empíricos. Todos os 10 participantes do GE responderam por e-mail no período de novembro a dezembro de 2018.

Depois desse grupo, foram convidados os participantes do GP para responder o mesmo instrumento de avaliação, sendo, desta vez, para avaliar a $2^{\mathrm{a}}$ versão do checklist, revisada a partir das respostas do GE. Nesse momento, durante a primeira quinzena de janeiro de 2019, a pesquisa foi realizada em um serviço de saúde que compõe a atenção básica do município de João Pessoa (Distrito Sanitário III/DS III). A escolha do serviço deu-se pelo fato de ser, a princípio, a porta de entrada dos usuários idosos ao serviço de saúde. Vale salientar que esse serviço faz parte do Sistema Único de Saúde (SUS), destituído de caráter privado, prestando assistência à comunidade, no nível primário e no secundário. Nesse serviço, existe um elevado perfil epidemiológico de idosos com alterações da audição e linguagem. Os 30 participantes do GP realizaram a avaliação do conteúdo do checklist versão 2 e, a partir de suas respostas, foi criada a $3^{\mathrm{a}}$ versão, resultado do trabalho de dissertação de mestrado.

Foi criado um instrumento para coleta dos dados (questionário de avaliação do checklist) composto por 12 perguntas: (1). São coerentes com as peculiaridades das pessoas idosas? (2). Contribui para reflexão da necessidade da avaliação fonoaudiológica? (3). Pode circular nos serviços de saúde para detectar a necessidade de encaminhar o idoso ao fonoaudiólogo? (4). O checklist é apropriado para rastrear as alterações de audição e linguagem da pessoa 
idosa? (5). As perguntas estão apresentadas de maneira clara e objetiva? (6). As perguntas apresentadas estão cientificamente corretas? (7). As perguntas estão bem estruturadas em concordância e ortografia? (8). A linguagem está adequada ao nível de conhecimento do público-alvo (profissionais de saúde não fonoaudiólogos)? (9). O checklist propõe ao profissional de saúde não-fonoaudiólogo, rastrear as alterações de audição e linguagem em idosos? (10). O checklist aborda assuntos necessários para o encaminhamento ao profissional de fonoaudiologia, visando a promoção/recuperação da saúde do idoso? (11). Está adequado para ser utilizado como tecnologia de tomada de decisão, de forma eficaz e célere, auxiliando no encaminhamento do idoso para o fonoaudiólogo? (12). É viável? Sendo ainda disponibilizado um espaço para sugestões ou comentários.

Os dados foram categorizados em planilha digital no Microsoft Excel ${ }^{\circledR}$. Posteriormente, as variáveis foram analisadas de forma descritiva: a apresentação dos resultados foi realizada por estatística descritiva simples (frequência absoluta e percentual) e a discussão foi embasada em publicações relacionadas ao tema, a fim de verificar a associação entre a opinião dos experts e profissionais de saúde. A avaliação dos juízes experts e dos profissionais de saúde foi construída a partir de perguntas fechadas a respeito de informações contidas no checklist quanto a sua relevância e utilidade para profissionais não-fonoaudiólogos que atuam junto à pessoa idosa.

Para a validação do conteúdo do instrumento, foi utilizado o índice de validade do conteúdo (IVC) maior ou igual a 0,78. Este método é muito utilizado na área de saúde e mensura a proporção que os experts e os profissionais de saúde concordam sobre determinados aspectos do instrumento. No método, foi empregada uma escala do tipo Likert de pontuação sendo $1=$ discordo plenamente, $2=$ discordo em parte, $3=$ concordo em parte, $4=$ concordo plenamente (Arceno \& Scharlach, 2017; Elias et al., 2015). O índice foi calculado pela soma dos itens 3 ou 4 pelos juízes (experts e profissionais de saúde), dividido pelo total de respostas.

A partir dos resultados analisados, foi elaborada a terceira versão do Checklist para rastrear as alterações de a audição e linguagem, que segue para as demais etapas de validação.

\section{Resultados}

A amostra foi constituída por 40 profissionais de saúde sendo 10 doutores experts na área de audição e linguagem, desses: um do sexo masculino e nove do sexo feminino, com idades entre 27 e 62 anos. E 30 profissionais de saúde não-fonoaudiólogos atuantes na prática assistencial na atenção básica junto a idosos. Desses, foram incluídos: 10 agentes comunitários de saúde (ACS), um odontólogo, três assistentes sociais, dois farmacêuticos, dois fisioterapeutas, cinco técnicos de enfermagem, quatro enfermeiros, dois psicólogos e um médico, dentre eles, 24 mulheres e seis homens, todos com idades entre 22 e 61 anos.

A versão 1 do checklist foi construída com base nas informações de artigos científicos originais referentes à audição e à linguagem de idosos. Foi realizada uma busca no sentido de identificar as principais alterações de audição e linguagem para em seguida formular o questionário tomando o cuidado em ser claro, breve e de fácil compreensão e entendimento tanto ao profissional de saúde (a quem vai aplicar para encaminhar ao fonoaudiólogo) como para o idoso que vai ser entrevistado.

Na Tabela 1, é possível observar o índice de validação de conteúdo para cada uma das respostas de ambos os grupos participantes. 
Tabela 1 - Índice de validação do conteúdo (IVC) por perguntas do GE (grupo de experts) e do GP (grupo de profissionais de saúde não-fonoaudiólogos). João Pessoa, PB, 2019.

\begin{tabular}{lcc}
\hline IVC POR PERGUNTAS & GE & GP \\
\hline 1- São coerentes com as peculiaridades das pessoas idosas & 1 & 0.9 \\
2- Contribui para reflexão da necessidade da avaliação & 0.9 & 0.9 \\
3- Pode circular nos serviços de saúde para detectar a necessidade de encaminhar os idosos & 0.8 & 0.9 \\
& & \\
ao fonoaudiólogo & 0.8 & 0.9 \\
4- O checklist é apropriado para rastrear as alteraçães de audição e linguagem em idosos. & 0.9 & 0.9 \\
5- As perguntas estão apresentadas de maneira clara e objetiva & 1 & 0.9 \\
6- As perguntas apresentadas estão bem elaboradas & 1 & 0.9 \\
7- As perguntas são bem estruturadas em concordância e ortografia & 0.9 & 0.9 \\
8- A linguagem está adequada ao nível de conhecimento do público-alvo (profissionais de & & \\
saúde não fonoaudiólogos) & 1 & 0.9 \\
9- O checklist propõe ao profissional de saúde não fonoaudiólogo rastrear as alterações de & & \\
audição e linguagem em idosos & 0.9 & 0.9 \\
10- O checklist aborda os assuntos necessários pra o encaminhamento ao profissional de & & \\
fonoaudiologia, visando a promoção/recuperação da saúde do idoso & 0.9 & 0.9 \\
11- Está adequado para ser utilizado como tecnologia de tomada de decisão de forma eficaz & & \\
e célebre, auxiliando no encaminhamento do idoso para o fonoaudiólogo & 1 & 0.9 \\
12- É viável &
\end{tabular}

Fonte: Dados da pesquisa realizada pelos Autores.

Na Tabela 2, observam-se as respostas dos grupos quanto aos itens de avaliação do checklist.

Tabela 2 - Respostas dos experts e profissionais não-fonoaudiólogos quanto aos itens de avaliação do checklist. João Pessoa, PB,2019.

\begin{tabular}{|c|c|c|c|c|c|c|c|c|c|c|c|c|c|c|c|c|c|c|}
\hline \multirow{3}{*}{$\begin{array}{c}\text { Itens de } \\
\text { avaliação } \\
\text { do checklist }\end{array}$} & \multirow{3}{*}{$\begin{array}{l}\text { Pergunta } \\
\quad \mathrm{s}\end{array}$} & \multicolumn{8}{|c|}{ EXPERTS } & \multicolumn{9}{|c|}{ PROFISSIONAIS NÃO-FONOAUDIÓLOGOS } \\
\hline & & \multicolumn{2}{|c|}{$\begin{array}{c}\text { Discordo } \\
\text { Plenament } \\
\text { e }\end{array}$} & \multicolumn{2}{|c|}{$\begin{array}{l}\text { Discord } \\
\text { o } \\
\text { em parte }\end{array}$} & \multicolumn{2}{|c|}{$\begin{array}{l}\text { Concord } \\
\text { o } \\
\text { em parte }\end{array}$} & \multicolumn{2}{|c|}{$\begin{array}{c}\text { Concordo } \\
\text { plenament } \\
\mathrm{e}\end{array}$} & \multicolumn{3}{|c|}{$\begin{array}{c}\text { Discordo } \\
\text { plenament } \\
\mathrm{e}\end{array}$} & \multicolumn{2}{|c|}{$\begin{array}{l}\text { Discord } \\
\text { o } \\
\text { em parte }\end{array}$} & \multicolumn{2}{|c|}{$\begin{array}{l}\text { Concordo } \\
\text { em parte }\end{array}$} & \multicolumn{2}{|c|}{$\begin{array}{c}\text { Concordo } \\
\text { Plenamen } \\
\text { te }\end{array}$} \\
\hline & & $\mathrm{N}$ & $\%$ & $\mathrm{~N}$ & $\%$ & $\mathrm{~N}$ & $\%$ & & $\%$ & $\mathrm{~N}$ & & $\%$ & $\mathrm{~N}$ & $\%$ & $\mathrm{~N}$ & $\%$ & $\mathrm{~N}$ & $\%$ \\
\hline \multirow{3}{*}{$\begin{array}{l}\text { 1.Objetivo } \\
\text { do } \\
\text { instrumento }\end{array}$} & 1.1 & 0 & 0 & 0 & 0 & 4 & 40 & & 60 & 2 & &, 67 & 1 & $\begin{array}{c}3,3 \\
3\end{array}$ & 5 & $\begin{array}{c}16,6 \\
7\end{array}$ & 22 & 73,33 \\
\hline & 1.2 & 0 & 0 & 0 & 0 & 2 & 20 & & 80 & 1 & &, 33 & 1 & $\begin{array}{c}3,3 \\
3\end{array}$ & 8 & 26,6 & 20 & 66,67 \\
\hline & 1.3 & 0 & 0 & 1 & 10 & 2 & 20 & & 70 & 1 & & 33 & 1 & $\begin{array}{c}3,3 \\
3\end{array}$ & 2 & 6,67 & 26 & 86,7 \\
\hline \multirow{5}{*}{$\begin{array}{l}\text { 2.Estrutura } \\
\text { e } \\
\text { apresentaçã } \\
\text { o } \\
\text { do } \\
\text { instrumento }\end{array}$} & 2.1 & 0 & 0 & 2 & 20 & 4 & 40 & 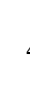 & 40 & 1 & & 33 & 1 & $\begin{array}{c}3,3 \\
3\end{array}$ & 5 & $\begin{array}{c}16,6 \\
7\end{array}$ & 23 & 76,7 \\
\hline & 2.2 & 0 & 0 & 1 & 10 & 1 & 10 & & 80 & 1 & &, 33 & 0 & 0 & 4 & $\begin{array}{c}13,3 \\
3\end{array}$ & 25 & 83,33 \\
\hline & 2.3 & 0 & 0 & 0 & 0 & 3 & 30 & & 70 & ( & & 0 & 2 & $\begin{array}{c}6,6 \\
7\end{array}$ & 8 & 26,6 & 20 & 66,67 \\
\hline & 2.4 & 0 & 0 & 0 & 0 & 5 & 50 & & 50 & ( & & 0 & 2 & $\begin{array}{c}6,6 \\
7\end{array}$ & 0 & 0 & 28 & 93,33 \\
\hline & 2.5 & 0 & 0 & 1 & 10 & 2 & 20 & & 80 & & &, 67 & 0 & 0 & 2 & 6,67 & 26 & 86,7 \\
\hline \multirow{4}{*}{$\begin{array}{l}\text { 3.Relevânci } \\
\text { a } \\
\text { do } \\
\text { instrumento }\end{array}$} & 3.1 & 0 & 0 & 0 & 0 & 4 & 40 & & 60 & & & 33 & 0 & 0 & 4 & $\begin{array}{c}13,3 \\
3\end{array}$ & 25 & 83,33 \\
\hline & 3.2 & 0 & 0 & 1 & 10 & 5 & 50 & & 40 & & & 33 & 1 & $\begin{array}{c}3,3 \\
3\end{array}$ & 5 & $\begin{array}{c}16,6 \\
6\end{array}$ & 23 & 76,66 \\
\hline & 3.3 & 0 & 0 & 1 & 10 & 3 & 30 & ( & 60 & & &, 33 & 1 & $\begin{array}{c}3,3 \\
3\end{array}$ & 3 & 10 & 25 & 83,33 \\
\hline & 3.4 & 0 & 0 & 0 & 0 & 3 & 30 & & 70 & & & 33 & 1 & $\begin{array}{c}3,3 \\
3\end{array}$ & 1 & 3,33 & 27 & 90 \\
\hline
\end{tabular}


O Quadro 1 apresenta comentários dos participantes sobre o checklist avaliado nas etapas 1 e 2.

Quadro 1 - Comentários dos experts e profissionais de saúde não-fonoaudiólogos sobre o checklist avaliado (versões 1 e 2).

\begin{tabular}{|c|c|c|}
\hline $\begin{array}{l}\text { VOLUNTÁRIO } \\
\text { DA PESQUISA }\end{array}$ & $\begin{array}{c}\text { GRUPO } \\
\text { PARTICIPANTE }\end{array}$ & COMENTÁRIOS SOBRE O CHECKLIST \\
\hline $\mathrm{V} 2$ & EXPERT & $\begin{array}{l}\text { “(...) Acredito que pode circular sim nos serviços de saúde, mas antes é } \\
\text { necessário, pelo menos, um treinamento das pessoas que terão acesso e utilizarão } \\
\text { esse Instrumento...”; “(...) O checklist propõe ao profissional de saúde não } \\
\text { fonoaudiólogo rastrear as POSSÍVEIS alterações de audição e linguagem em } \\
\text { idosos”; "O checklist aborda os assuntos necessários às duas áreas específicas da } \\
\text { fonoaudiologia para que seja realizado o devido encaminhamento, para o } \\
\text { profissional de fonoaudiologia, visando a promoção/recuperação da saúde do } \\
\text { idoso”. }\end{array}$ \\
\hline V6 & EXPERT & $\begin{array}{c}\text { "Achei muito interessante o instrumento, com relevância científica tanto à } \\
\text { Fonoaudiologia, com relação às áreas de linguagem e audição, quanto à } \\
\text { Gerontologia. O instrumento está claro, bem objetivo e, sem dúvida, será de } \\
\text { importância grande à ciência". }\end{array}$ \\
\hline V19 & $\begin{array}{c}\text { PROFISIONAL } \\
\text { (FISIOTERAPEUTA) }\end{array}$ & $\begin{array}{l}\text { "Acho extremamente importante a utilização de um checklist como esse, porque } \\
\text { no mínimo trabalhamos com a promoção em relação à audição e linguagem. } \\
\text { Trabalho a mais de } 20 \text { anos com a pessoa idosa e escuto queixas recorrentes em } \\
\text { relação a esses problemas principalmente com pacientes neurológicos" }\end{array}$ \\
\hline V27 & $\begin{array}{l}\text { PROFISSIONAL } \\
\text { (PSICÓLOGO) }\end{array}$ & $\begin{array}{c}\text { "Entendo como um instrumento essencial para identificação e diagnóstico } \\
\text { preliminar necessário na identificação de sintomas que apontam com clareza no } \\
\text { desenvolvimento desta pesquisa para a elaboração do plano da assistência aos } \\
\text { pacientes que apresentam necessidade do tratamento" }\end{array}$ \\
\hline V35 & $\begin{array}{l}\text { PROFISSIONAL } \\
\text { (ENFERMEIRA) }\end{array}$ & $\begin{array}{l}\text { "Achei um projeto de extrema necessidade para lidar com os idosos e detectar } \\
\text { dificuldades que até então não são possíveis serem notadas. Esse questionário } \\
\text { facilitaria para que nós não fonoaudiólogos percebêssemos estas dificuldades } \\
\text { dos idosos” }\end{array}$ \\
\hline
\end{tabular}

Fonte: Dados da pesquisa realizada pelos Autores.

O Quadro 2 apresenta a versão 3 do checklist. 
Quadro 2 - Checklist (versão final) apresentado como produto de dissertação de mestrado profissional em Gerontologia. João Pessoa, PB, 2019.

\begin{tabular}{|c|c|c|}
\hline $\begin{array}{lll}\text { NOME: } & \text { SEXO: ( ) M ( ) F } \\
\text { DATA } & \text { DE }\end{array}$ & & NASC.: \\
\hline \multicolumn{3}{|l|}{ QUEIXA: } \\
\hline \multicolumn{3}{|l|}{ PROFISSIONAL: } \\
\hline REGISTRO: & & DATA: \\
\hline ASPECTOS DA AUDIÇÃO & $\mathrm{S}$ & $\mathrm{N}$ \\
\hline \multicolumn{3}{|l|}{ 1-Você tem dificuldade de entender em ambiente com barulho? } \\
\hline \multicolumn{3}{|l|}{ 2-Você tem incômodo quando ouve sons muito alto? } \\
\hline \multicolumn{3}{|l|}{ 3-Você tem percebido sua audição diminuir com o passar dos anos? } \\
\hline \multicolumn{3}{|l|}{ 4- Você tem deixado de participar de atividades sociais? } \\
\hline \multicolumn{3}{|l|}{ 5-Você tem dificuldade de escutar televisão/rádio ou pessoas que falam baixo? } \\
\hline \multicolumn{3}{|l|}{ 6-Você pede para as pessoas repetirem uma pergunta ou conversa? } \\
\hline \multicolumn{3}{|l|}{ 7-Você tem ouvido algum barulho nos ouvidos que outras pessoas não escutam? } \\
\hline ASPECTOS DA LINGUAGEM & $\mathrm{S}$ & $\mathrm{N}$ \\
\hline \multicolumn{3}{|l|}{ 1- Você tem esquecimentos durante uma conversa? } \\
\hline \multicolumn{3}{|l|}{ 2-Você tem dificuldade para lembrar histórias ou nomes? } \\
\hline \multicolumn{3}{|l|}{ 3-Você tem dificuldade de acompanhar a lógica ou raciocínio de uma conversa? } \\
\hline \multicolumn{3}{|l|}{ 4-Você tem dificuldade de prender a atenção em uma conversa? } \\
\hline \multicolumn{3}{|l|}{ 5-Você tem dificuldade em formular frases durante uma conversa? } \\
\hline \multicolumn{3}{|l|}{ 6-Você tem dificuldade de ser compreendido(a) quando fala? } \\
\hline 7-Você tem ouvido as pessoas dizerem que não entendem você durante uma conversa? & & \\
\hline
\end{tabular}

Fonte: Autores.

\section{Discussão}

De maneira geral, um checklist segue como parte de uma lista de verificação que pode aparecer na forma de questões ou ações na sua utilização (Azevedo et al., 2015; Borges et al., 2016). Foi pensando nisso e na necessidade de encaminhamento de idosos a fonoaudiólogos por parte dos profissionais de saúde que lidam diretamente com o idoso, que surgiu o interesse em elaborar esse instrumento para possível encaminhamento, na intenção de uma avaliação mais específica, promovendo a promoção e recuperação da audição e linguagem de idosos.

Considera-se o checklist como uma ferramenta recomendada por organizações e estudiosos da área. Estudos têm comprovado sua eficácia, sendo a prática importante e recentemente introduzida na área da saúde (Purim et al., 2019; Gomes, Santos, Machado, \& Treviso, 2016). A análise da utilização do checklist permite identificar problemas e fatores associados ao seu efetivo uso para possibilitar a incorporação da tecnologia, visando aos resultados positivos para a segurança do paciente (Ribeiro, Quites, Bredes, Sousa, \& Alves, 2017; Nascimento, Batista, Rocha, \& Vasconcelos, 2015). O uso do checklist, mostra a terceiros não só a responsabilidade e seriedade, mas ajuda também a criar uma amostra de disciplina e facilitar na concentração durante todos os procedimentos. A prática do uso do instrumento proporciona uma melhor qualidade na assistência, auxiliando os profissionais de saúde no rastreamento das alterações de audição e linguagem do idoso, prestando os cuidados necessários em todo o processo pré-avaliativo (Comiotto, Kappaun, \& Cesa, 2018; Oliveira, Abreu, \& Almeida, 2017). 
O uso de checklist tem sido foco de estudos sobre sua forma de uso nas diferentes fases, na rotina de trabalho, considerando que seja uma ferramenta que permite a checagem de itens importantes que poderiam ser esquecidos pela equipe multiprofissional (Silva et al., 2016). No Brasil, existem poucos estudos que apresentam métodos de trabalho no processo de implantação e de preenchimento correto de instrumentos desse tipo, bem como sua importância para a redução de incidentes (Ribeiro et al., 2017). Esses instrumentos de verificação podem transformar a forma como o conhecimento é colocado em prática, além de constituir recurso básico e de custo baixíssimo aos serviços de saúde (Alpendre et al., 2017).

Conforme a Tabela 1, a relevância avaliada pelos participantes caracterizou um índice da validação de conteúdo (IVC) maior que 0,78, conforme recomendação (Arceno \& Scharlach, 2017; Elias et al., 2015). O cálculo do IVC foi apresentado entre 0,8 e 1 para os experts, e de 0,9 para os profissionais de saúde para todos os itens. Em ambas as etapas. Verifica-se uma constante para todas as respostas dos itens, ou seja, concordância de que é possível rastrear as alterações de audição e linguagem de idosos por meio do instrumento proposto. Pode-se afirmar que o checklist aborda os assuntos necessários, está adequado para ser utilizado e é viável. Por isso, inclusive, não foi possível calcular o Qui Quadrado e p-valor nos dois grupos (GE e GP), já que houve concordância nas respostas "concordo em parte" e "concordo plenamente" de acordo com a escala Likert do instrumento de avaliação, sendo que este último chega a $80 \%$ no grupo dos experts.

No que se refere aos comentários dos experts, as sugestões para melhorar a proposta do checklist foram acatadas de forma que melhorou a comunicação de ambas as partes (profissionais e idosos). Em ambos os grupos, apareceram elogios para o checklist, recomendando-o, e ressaltando sua importância, como se observa na Figura 1.

A promoção da saúde refere-se ao cuidado integral da saúde e implica em ações de promoção da saúde. Saúde é um direito humano fundamental reconhecido por todos os foros mundiais e em todas as sociedades. Promover a saúde é promover a qualidade de vida (Pereira, Souza, Carneiro, \& Sarges, 2014; Malta et al., 2016). A promoção da saúde é o resultado de um conjunto de fatores sociais, econômicos, políticos e culturais, coletivos e individuais, que se combinam de forma particular em cada sociedade e em conjunturas específicas, resultando em sociedades mais ou menos saudáveis (Purim et al., 2019, Pereira et al., 2014). A promoção da saúde refere-se às ações sobre os condicionantes e determinantes sociais da saúde, dirigidas a impactar favoravelmente a qualidade de vida (Amaya, Paixão, Sarquis, \& Cruz, 2016; Janini, Bessler, \& Vargas, 2015).

Os participantes do estudo (experts e profissionais de saúde não-fonoaudiólogos) levantaram sugestões e elogios à proposta oferecida. Dos dez experts, todos apresentaram considerações construtivas na melhoria da linguagem ou na forma de abordar as questões do checklist. Quanto aos profissionais de saúde, dos 30 entrevistados, sete comentaram de forma satisfatória todo o conteúdo da segunda versão do checklist. No momento da avaliação, todos demonstraram satisfação em responder o instrumento de pesquisa para obter posteriormente respostas para o serviço, principalmente por parte dos ACS que tiveram bastante cuidado de ler e entender o conteúdo. Quem mais respondeu ao instrumento de pesquisa foram os ACS, técnicos de enfermagem e enfermeiros pela disponibilidade de tempo e interesse.

A intenção do checklist foi despertar nos profissionais de saúde não- fonoaudiólogos que trabalham junto à pessoa idosa sobre a necessidade de encaminhar ao fonoaudiólogo. A partir dele, é realizado um rastreamento referente às alterações da audição e linguagem, visto que o mesmo segue como uma folha de verificação, podendo ser aplicado por qualquer profissional. É um instrumento simples, de rápida aplicação, conforme preconizado na literatura (Pereira et al., 2014; Malta et al., 2016). 
O processo de construção do checklist contou com uma pesquisa metodológica de abordagem quantitativa, na qual foi possível observar a relevância, nos últimos dez anos, de progressão dos estudos sobre a comunicação de idosos nos âmbitos da audição e linguagem. Considerando que a perda ou deficiência auditiva decorrente da idade (presbiacusia) tem papel importante e significativo para a dificuldade de compreensão e expressão da linguagem, trazendo prejuízo no convívio social, estresse e depressão, e que a falha na memória, seja ela de caráter degenerativo ou transitório, aponta também como um fator causal para uma comunicação ineficaz, fez pensar nestas duas áreas da fonoaudiologia (audição e linguagem) para a elaboração deste instrumento (checklist) (Janini et al., 2015).

Portanto, o checklist (Figura 2) pode ser aplicado pelos profissionais de saúde que trabalham com idosos em serviços de saúde para rastrear as alterações de audição e linguagem e encaminhar ao fonoaudiólogo no intuito de uma avaliação mais específica dando-lhe a oportunidade de qualidade de vida. Ressalta-se a importância do instrumento e, para isso, o treinamento dos profissionais não-fonoaudiólogos, para o uso adequado e aplicação correta do checklist. Ressalta-se que existe o interesse em um curso de doutorado, aprofundar o estudo do checklist e realizar as demais etapas de validação, inserindo, inclusive, escores no instrumento, tornando-o mais objetivo e preciso.

\section{Conclusão}

A intenção do checklist foi despertar nos profissionais de saúde não-fonoaudiólogos que trabalham junto à pessoa idosa sobre a necessidade de encaminhar ao fonoaudiólogo. A partir dele, é realizado um rastreamento referente às alterações da audição e linguagem, visto que o mesmo segue como uma folha de verificação, podendo ser aplicado por qualquer profissional. É um instrumento simples, de rápida aplicação, conforme preconizado na literatura.

O processo de construção do checklist contou com uma pesquisa metodológica de abordagem quantitativa, na qual foi possível observar a relevância, nos últimos dez anos, de progressão dos estudos sobre a comunicação de idosos nos âmbitos da audição e linguagem.

Considerando que a perda ou deficiência auditiva decorrente da idade (presbiacusia) tem papel importante e significativo para a dificuldade de compreensão e expressão da linguagem, trazendo prejuízo no convívio social, estresse e depressão, e que a falha na memória, seja ela de caráter degenerativo ou transitório, aponta também como um fator causal para uma comunicação ineficaz, fez pensar nestas duas áreas da fonoaudiologia (audição e linguagem) para a elaboração deste instrumento (checklist).

Foi possível, portanto, construir o instrumento. Entretanto agora é necessário o treinamento dos profissionais nas unidades básicas, para que possam fazer a correta aplicação, rastreando as possíveis alterações de audição e linguagem para gerar a demanda de atendimento em fonoaudiologia. Com isso, ganharão os profissionais, com a ampla divulgação da atuação fonoaudiológica, bem como os idosos, que serão direcionados ao atendimento especializado.

Como sugestão de trabalhos futuros, sugere-se que este checklist aqui produzido seja aplicado pelas diversas categorias profissionais de saúde, inclusive as de nível médio, para o rastreio de alterações de audição e de linguagem em idosos senescentes e que os resultados sejam apresentados a fim de confirmar a aplicabilidade do instrumento. Sugere-se que o instrumento seja utilizado especialmente por profissionais de saúde que não o fonoaudiólogo, nas UBS, por médicos, enfermeiros, ACS's e outros profissionais que atendam a pessoa idosa, a fim de que cada vez mais se tenha um olhar holístico sobre a saúde do idoso. 


\section{Referências}

Alpendre, F. T., Cruz, E. D. A., Dyniewicz, A. M., Mantovani, M. F., Silva, A. E. B. C., \& Santos, G. S. (2017). Cirurgia segura: validação de checklist pré e pós-operatório. Rev. Latino-Am. Enfermagem, 25, e2907.

Amaya, M. R., Paixão, D. P. S. S., Sarquis, L. M. M., \& Cruz, E. D. A. (2016). Construção e validação de conteúdo de checklist para a segurança do paciente em emergência. Revista Gaúcha de Enfermagem, 37, e68778.

Arceno, R. S., \& Scharlach, R. C. (2017). Teste de fala comprimida em idosos. CoDAS, 29(5), e20160243.

Azevedo, M. M., Santos, S. N., \& Costa, M. J. (2015). Desempenho de idosos com adaptação binaural x monoaural em testes de fala no silêncio e no ruído. Rev. CEFAC, 17(2), 431-438.

Borges, M. G. S., Labanca, L., Couto. E. A. B., \& Guarisco, L. P. C. (2016). Correlations between the audiological evaluation and cognitive screening in the elderly. Rev. CEFAC, 18(6), 1285-1293.

Comiotto, G. S., Kappaun, S., \& Cesa, C. C. (2016). Conhecimento dos profissionais da área da saúde acerca da comunicação suplementar e alternativa em instituições de longa permanência para idosos. Rev. CEFAC, 18(5), 1161-1168.

Elias, A. C. G. P., Schmidt, D. R. C., Yonekura, C. S. I., Dias, A. O., Ursi, E. S., Silva, R. P. J., \& Feijo, V. B. E. L. (2015). Evaluation of the adherence to the safe surgery checklist at the public university. Rev. SOBECC, 20(3), 128-133.

Estrela, C. (2018). Metodologia Científica: Ciência, Ensino, Pesquisa. Artes Médicas.

Giannattasio, M. G., \& Taniguchi, F. P. (2016). Avaliação da segurança do paciente em cirurgia cardíaca de um hospital público. Rev. SOBECC, 21(3), 125-131.

Gomes, C. D. P. P., Santos, A. A., Machado, M. E., \& Treviso, P. (2016). Percepção de uma equipe de enfermagem sobre a utilização do checklist cirúrgico. Rev. SOBECC, 21(3), 140-145.

Janini, J. P., Bessler, D., \& Vargas, A. B. (2015). Educação em saúde e promoção da saúde: impacto na qualidade de vida do idoso. Saúde debate, 39(105), 480-490.

Kim, G., Na W., Kim G., Han, W., \& Kim, J. (2016). The development and standardization of self-assessment for hearing screening of the elderly. Clinical Interventions in Aging, 11(1), 787-795.

Malta, D. C., Morais Neto, O. L., Silva, M. M. A., Rocha, D., Castro, A. M., Reis, A. A. C., \& Akerman, M. (2016). Política Nacional de Promoção da Saúde (PNPS): capítulos de uma caminhada ainda em construção. Ciênc. saúde coletiva, 21(6), 1683-1694.

Nascimento, R. A. S. A., Batista, R. T. S., Rocha, S. V., \& Vasconcelos, L. R. C. (2015). Prevalência e fatores associados ao declínio cognitivo em idosos com baixa condição econômica: estudo MONIDI. J.Bras. de Psiquiatria, 64(3), 187-192.

Oliveira, A. C., Abreu, A. R., \& Almeida, S. S. (2017). Implementação do checklist de cirurgia segura em um hospital universitário. Enferm. Foco, 8(4), 14-18.

Pereira, E. E. B., Souza, A. B. F., Carneiro, S. R., \& Sarges, E. S. N. F. (2014). Funcionalidade global de idosos hospitalizados. Rev. Bras. Geriatr. Gerontol, 17(1), 165-176.

Porto, K. L. H. (2014). A segurança do paciente na utilização do checklist. Rev. Enfermagem Revista, 17(2), $103-115$.

Purim, K. S. M., Gonçalves, C. G., Binotto, L., Groth, A. K., Aranha Júnior, A. A., Chibata, M., \& Tsumanuma, F. K. (2019). Checklist de segurança no ensino de cirurgia ambulatorial. Rev. Col. Bras. Cir., 46(3), e20192197.

Ribeiro, H. C. T. C., Quites, H. F. O., Bredes, A. C., Sousa, K. A. S., \& Alves, M. (2017). Adesão ao preenchimento do checklist de segurança cirúrgica. Cad. Saúde Pública, 33(10), e00046216.

Silva, S. G., Nascimento, E. R. P., Hermida, P. M. V., Sena, A. C., Klein, T. C. R., \& Pinho, F. M. (2016). Checklist para passagem de plantão de pacientes em pós-operatório imediato na admissão em terapia intensiva. Enferm. Foco, 7(1), 13-17. 